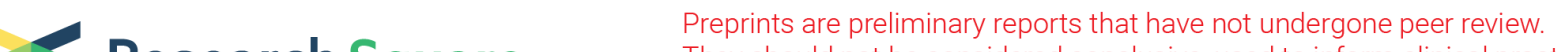 Research Square They should not be considered conclusive, used to inform clinical practice, or referenced by the media as validated information.
}

\section{Relationship between enlarged perivascular space in hippocampus and P300 event-related potential in patients with acute lacunar stroke}

\section{Shan Huang}

Dalian Medical University

\section{Zhixiang Zhang}

Changzhou NO.2 People's Hospital

\section{Yuqing Mei}

Changzhou NO.2 People's Hospital

\section{Kuankuan Huang}

Changzhou NO.2 People's Hospital

\section{Yiping Feng}

Changzhou NO.2 People's Hospital

\section{Xia Gu}

Changzhou NO.2 People's Hospital

Qiaoyang Zhang

Changzhou NO.2 People's Hospital

Min Zhang ( $\square$ zhangjueming11@163.com )

Changzhou NO.2 People's Hospital Affiliated to Nanjing Medical University https://orcid.org/00000003-3568-7351

\section{Wenwei Yun}

Changzhou NO.2 People's Hospital

\section{Research article}

Keywords: Hippocampus enlarged perivascular space, P300 event-related potential, Acute lacunar stroke, Verbal fluency function

Posted Date: January 15th, 2021

DOI: https://doi.org/10.21203/rs.3.rs-145936/v1

License: (c) (1) This work is licensed under a Creative Commons Attribution 4.0 International License.

Read Full License 


\section{Abstract \\ Background}

This study aimed to investigate the relationship between enlarged perivascular space in hippocampus $(\mathrm{H}-$ EPVS) and P300 event-related potential (ERP) in patients with acute lacunar stroke.

\section{Methods}

Patients with acute lacunar stroke hospitalized in Changzhou No.2 people's Hospital from February 2019 to July 2020 were collected. All the patients performed the P300 auditory oddball task and a full set of cognitive function tests. We recorded P300 latency and amplitude by EMG evoked potential instrument. H-EPVS showed round, oval and linear structures on MRI T2-weighted images of hippocampus, with clear boundaries and consistency in the direction of the perforating arterioles. Dividing the total number of $\mathrm{H}$ EPVS $\geq 7$ into extensive H-EPVS group $(n=61)$ and non-extensive H-EPVS group $(n=53)$. ROC curve was used to analyze the relationship between P300 ERP and cognitive function of extensive H-EPVS in patients with acute lacunar stroke.

\section{Results}

A total of 114 patients with acute lacunar stroke were included, and 61 (53.5\%) patients were found to have extensive H-EPVS. The P300 latency in patients with extensive H-EPVS( mean and SD, $393.61 \pm$ 30.36) was significantly higher than in non-extensive H-EPVS (mean and SD, $356.4 \pm 19.59 \mathrm{~ms}, t=-7.870$, $P<0.01)$. There was no significant difference in P300 amplitude between the two groups $(P>0.05)$. In addition, The cognitive function (the field of verbal fluency) in patients with extensive H-EPVS (mean and SD, $-0.41 \pm 0.50$ ) was significantly lower than in non-extensive H-EPVS (mean and SD, $0.47 \pm 0.54, t=$ $9.017, P<0.01)$. Further analysis shows that in patients with acute lacunar stroke, P300 latency was positively associated with H-EPVS counts $(r=0.726, P=0.000)$. H-EPVS counts was negatively associated with cognitive function score (the field of verbal fluency) $(r=-0.705, P=0.000)$. ROC curve analysis showed that the index of cognitive impairment diagnosed by P300 in patients with acute lacunar stoke was 0.796 .

\section{Conclusion}

Extensive H-EPVS has a high incidence in patients with acute lacunar stroke. The P300 latency may be used as an early indicator to evaluate the verbal fluency function of extensive H-EPVS patients with acute lacunar stroke.

\section{Background}


The perivascular spaces (PVS) is a potential space around the perforating branch of the brain, the products of brain metabolism can be cleared through this space [1]. At present, it is believed that the PVS visible in MRI is enlargement perivascular spaces (EPVS), and diameter is usually $<3 \mathrm{~mm}$.

Previous studies have found that EPVS in the basal ganglia (BG-EPVS) and central semioval (CSO-EPVS) is the most common area. The occurrence of EPVS in hippocampus (H-EPVS) has been paid more and more attention. The hippocampal vascular system is the basis for maintaining the normal function of the hippocampus. However, the diameter of the artery that directly supplies the hippocampus and its branches is very small [2,3]. Physiological (aging) or pathological (stroke, Alzheimer' s disease) cases, the blood vessels in the hippocampus may be more easily affected by decreased blood perfusion and hypoxia, resulting in hippocampal neuronal dysfunction [3].

The hippocampal formation is closely related to cognitive function. P300 may be an important index to evaluate the hippocampus function. P300 event-related potential (ERP) is considered the most valuable neurophysiological index to reflect the process of individual cognitive processing [4]. P300 (or 'P3b') is related to the information processing and contextual memory updating when brain performing tasks [5]. Unfortunately, the relationship between H-EPVS and P300 in patients with acute lacunar stroke is still unclear.

In this study, we aim to understand the incidence of H-EPVS in patients with lacunar stroke and we want to determine whether P300 ERP is associated with H-EPVS cognitive function.

\section{Methods}

\section{Patients}

A total of 137 patients with acute lacunar stroke hospitalized in Changzhou No.2 people's Hospital from February 2019 to July 2020 were collected. Inclusion criteria (1) Patients with first lacunar stroke; (2) age between 50 and 80 years at the inclusion; (3) Hearing is normal; (4) No history of dementia. (6) Be able to complete the MRI examination of the skull and P300 ERP detection. Exclusion criteria: (1) Known history of stroke, epilepsy, depression (excluded by self-rating depression scale) or other neurological or mental disorders (excluded by clinical evaluation and known history); (2) hearing loss and inarticulate; (3) weakness of the right limb. (4) Cognitive disorders that may be caused by other diseases (e.g. cancer, hepatorenal insufficiency, anemia, hypothyroidism). (5) Take drugs that affect cognitive function, such as anti-anxiety drugs.

Among these subjects, 3 subjects had hearing loss, 12 subjects had weakness of the right limb, and 8 subjects had inarticulate. Finally, 114 subjects performed P300 ERP and MRI examination and neuropsychological assessment. Dividing the total number of H-EPVS $\geq 7$ into extensive H-EPVS group ( $n$ = 61) and non-extensive H-EPVS group $(n=53)$. Physiologically right-handed. This study was approved by the Instituted Ethics Committees of the Changzhou No.2 people's Hospital Affiliated to Nanjing Medical University (NO.2018-KY032-01). Informed consent was obtained from all participants. 


\section{EPVS and other markers}

We used GE 3.0T MR (Discovery MR750, USA) imaging system scan the T1WI, T2WI and T2 Flair sequence of all subjects. EPVS showed round, oval and linear structures with clear boundaries consistency in the direction of perforating arterioles (due to differences in location and section) on MRI. T1WI and FLAIR sequences showed low signal intensity, T2WI showed high signal intensity, and was the same as cerebrospinal fluid signal. EPVS usually occurs in BG, CSO, hippocampus and brainstem. Visual quantification was used to count the largest number of EPVS in unilateral BG and CSO plane [6] : grade 0, was non-EPVS; grade 1 was EPVS $\leq 10$; grade 2 was EPVS 11 20; grade 3 was EPVS 21 40; grade 4 was EPVS >40. Grade 0 1 was defined as mild EPVS, Grade 2 4 was defined as moderate -severe EPVS [6] (Fig1 B C). We calculate total count of left and right hippocampus, H-EPVS $\geq 7$ was defined as extensive H-EPVS, H-EPVS 7 was defined as non-extensive H-EPVS [7, 8] (Fig1 A). We only calculate the maximum diameter $<3 \mathrm{~mm}$ EPVS, because the EPVS of $>3 \mathrm{~mm}$ may have different pathogenesis.

The white matter hyperintensities $(\mathrm{WMH})$ was the speckled or patchy high signal changes of the paraventricular or deep white matter on the FLAIR sequence. Fazekas score $\leq 2$ was defined as mild WMH, Fazekas score $\geq 3$ as moderate- severe WMH [9] (Fig1 D). Lacunar infarction usually shows marginal high signal intensity on T2WI sequence with a diameter of $\geq 3 \mathrm{~mm}$.

\section{Cognitive assessment}

All subjects were evaluated by the same physician trained by the formal unified neuropsychological scale in the same environment. The test scale includes overall cognitive function, memory, information processing speed, executive function and verbal fluency function. Convert the each test scores into standardized Z-score (individual test score - mean test score) / standard deviation [10]. Finally, the test $Z$ score of each cognitive domain is averaged, and the compound Z-score of the cognitive domain is obtained. The higher the Z-score, the better the performance.

The test scale includes: (1) Overall cognitive function: Montreal cognitive assessment (MoCA) has been widely used in vascular cognitive impairment and Mild cognitive impairment screening. Compared with mini-mental state examination (MMSE), it has higher sensitivity and specificity [11]. (2) Memory Z-score: Auditory word learning test (AVLT), Digit span test (DSهforward, $Z=(Z / A V L T$ immediate recall $+Z$ / AVLT delayed recall $+Z$ Z AVLT recognition $+Z$ / DS forward) / 4. (3) Information processing speed Z-score: Symbol digit modalities test (SDMT), Trail making test-A(TMT-A), Stroop color-word test-A(SCWT-A), Stroop color-word test-B(SCWT-B), Z= (Z/SDMT+Z/TMT-A+ average Z/ SCWT-A + SCWT-B completion time) / 3. (4) Executive function $Z$-score: SCWT interference score = SCWT completion time (SCWT-A+ SCWT-B) average completion time, TMT interference times=TMT-B-TMT-A, DS backward, $Z=$ (Z/ SCWT interference score $+Z /$ TMT interference times $+Z /$ DS backward) / 3. (5) Verbal fluency $Z$-score: Semantic verbal fluency, Phonemic verbal fluency, $Z=(Z /$ Semantic verbal fluency $+Z /$ Phonemic verbal fluency) / 2 [12]. 
The P300 wave was generated from the keypoint EMG evoked potential instrument produced by Dandy Company. The reference electrode was placed in the posterior mastoid of both ears, the recording electrode was placed at the $\mathrm{Cz}$ point, and the ground electrode was placed in the center of the forehead. It was suitable for scrub to reduce the resistance of each electrode to less than $5 \mathrm{~K} \Omega$, and the sensitivity was $5 \mu \mathrm{V}$. Auditory Oddball mode was selected, which consists of two different frequencies of sound. One was target stimulus, also known as treble stimulus, the occurrence rate was $20 \%$. The other was nontarget stimulus, also known as bass stimulus, the occurrence rate was $80 \%$. The two stimuli occur irregularly alternately, the interval of sound stimulation was $1.5 \mathrm{~s}$, superimposed 200 times. Keystroke responses to Target stimuli is monitored when the patients stays quiet and with closed eyes. The reaction time and hit rate of the subjects were recorded, the test was repeated twice, and the average value was taken.

In this study, basic waveform on the $\mathrm{Cz}$ points were recorded, the P300 latency (the straightline distance from the start of stimulation to the peak of the maximum P300 amplitude) and amplitude (the vertical distance from the baseline to the peak of P300) were analyzed in both groups.

\section{Statistical analysis}

H-EPVS was classified as extensive H-EPVS and non-extensive H-EPVS according to H-EPVS counts ( $\geq$ 7H-EPVS). To study the risk factors associated with H-EPVS classification (non-extensive and extensive H-EPVS), we first conducted a univariate analysis to associate H-EPVS classification with demographic information, VRF, P300 ERP, neuropsychological scale evaluation and other cerebral small vascular disease (CSVD) markers. Pearson's $\chi^{2}$ was used for the comparison of categorical variables, and MannWhitney $U$ test or $t$ test was used for continuous variables (Table 1). Second, Spearman correlation analysis was used to analyze the correlation between information processing speed $Z$-score, speech fluency Z-score, P300 latency and H-EPVS counts (Fig 2). After adjusting the covariates of age, sex and education years, further analysis the relationship between H-EPVS count and P300 latency. The results were expressed in terms of $\beta$ and $95 \%$ confidence interval (Table 2). Finally, receiver operating characteristic (ROC) curves was generated, and analyze the specificity and sensitivity of P300 latency in predicting cognitive impairment with extensive H-EPVS in patients with acute lacunar stroke.

\section{Results}

\section{General characteristics between extensive and non-extensive H-EPVS}

A total of 114 patients with lacunar stroke were included, and 61 (53.5\%) patients were found to have extensive H-EPVS. The age in extensive H-EPVS group (mean=67.18year, S. D. =6.87) was significantly higher than non-extensive H-EPVS group (mean= 64.09 year, S. D. $5.87, t=-2.558, P<0.05$ ). There was no significant difference in gender, education years, hpertension, diabetes, moderate-severe BG-EPVS, moderate-severe CSO-EPVS and moderate- severe WMH, lacunar infarction between two groups $(P>0.05)$ (Table 1). 
The information processing speed Z-score and Verbal fluency Z-score in extensive H-EPVS group were lower than non-extensive H-EPVS group $(P<0.01)$. The two subscales AVLT immediate recall and delayed recall $Z$-score reflecting memory function in the extensive H-EPVS group were also lower than those in the non-extensive H-EPVS group, but there was no significant difference in the $Z$ score of memory function and executive function between the two groups $(P>0.05)$ (Table 1$)$.

\section{P300 ERP resultsbetween extensive and non-extensive H-EPVS}

The P300 latency in patients with extensive H-EPVS( mean and SD, 393.61 \pm 30.36 ) was significantly higher than in non-extensive H-EPVS (mean and SD, $356.4 \pm 19.59 \mathrm{~ms}, t=-7.870, P<0.01$ ). There was no significant difference in P300 amplitude between two groups (mean P300 amplitude in extensive H-EPVS group $=8.42 \mu$ V, S.D. $=2.32$; mean P300 amplitude in non-extensive H-EPVS group = $8.93 \mu$ V, S.D. $=2.41$; $t=1.157, P>0.05$, Table 1$)$

\section{Spearman correlation Analysis of H-EPVS count}

H-EPVS count was negatively correlated with verbal fluency $Z$-score $(r=0.705, P=0.000)$ and positively correlated with P300 latency $(r=0.726, P=0.000)$. (Fig 2a, 2b).

\section{P300 latency and verbal fluency function}

P300 latency was negatively correlated with Verbal fluency Z-score $(r=-0.515 P=0.000$, Fig 2c)

\section{Linear regression Analysis}

In the multivariate regression analysis, H-EPVS count remained significantly correlated with the P300 latency $(\beta=4.659,95 \% \mathrm{Cl} 3.870$ to 5.448$)$ after adjusting by sex, age, education years (Table 2 ).

\section{ROC analysis predictive of H-EPVS cognitive function}

ROC curve analyses (Fig 3) were used to identify that the area under the curve (AUC) for the prediction of verbal fluency function with extensive H-EPVS patients using P300 latency was $0.796(95 \% \mathrm{Cl} 0.711$ $0.881, P<0.01)$.

\section{Discussion}

This study found that the incidence of extensive H-EPVS was higher in patients with lacunar stroke. P300 latency was positively associated with H-EPVS counts. Extensive H-EPVS has a high incidence in patients with acute lacunar stroke. The P300 latency may be used as an early indicator to evaluate the verbal fluency function of extensive H-EPVS patients with acute lacunar stroke.

It was reported that the incidence of H-EPVS in healthy people was $36.4-77 \%$, and the incidence of $\mathrm{H}-$ EPVS in stroke people was as high as $84 \%[13,14]$. Blood-brain barrier (BBB) is a unique structure in brain microcirculation, endothelial cells play an important role in maintaining tissue perfusion pressure and 
hemodynamics [15]. The endothelial cells damage of hippocampal artery leads to the increase of bloodbrain barrier permeability, the metabolite clearance disturbance in hippocampal artery and the interstitial fluid circulation disturbance will form H-EPVS [16]. This study found that age was risk factors for extensive H-EPVS, which was consistent with previous studies [7, 14]. Related studies found that WMH, lacunar infarction, BG-EPVS and CSO-EPVS was associated with H-EPVS [14, 17]. However, no association with extensive H-EPVS was found in this study.

At present, the research conclusions on H-EPVS and cognitive performance are not consistent. A study based on the patients with hypertension shows H-EPVS was related to worse verbal reasoning ability [7]. In contrast, no correlation was found between H-EPVS count and cognitive performance with dementia risk in healthy elderly and community populations $[14,17]$. We found that the information processing speed of extensive H-EPVS was lower than non-extensive H-EPVS, but no correlation was found between $\mathrm{H}$-EPVS count and information processing function. In addition, H-EPVS count was positively correlated with delayed P300 latency. P300 is a component that reflects the cognitive process, and the P300 latency reflects the brain recognizes information speed [5]. The origin of P300 in the brain is still not completely clear. It may represents the sum of complex activities of different generators in the limbic system and related regions of the cerebral cortex, and each generator may represent different cognitive performance [18]. Hippocampus as an important part of the marginal-cortical network, it is involved in the activity of the generator $[18,19]$. Previous research found P300 disappeared in patients with bilateral hippocampal lesions, P300 may reflect the hippocampal function damage. Scalp recording potentials involve related areas of the hippocampus and cerebral cortex [20]. H-EPVS was closely related to vascular neural unit dysfunction. Vascular neural units can dynamically reflect the synaptic activity and metabolism of neurons [21]. This may be due to extensive H-EPVS damage to the neuronal circuits of the hippocampus and medial temporal lobe as well as other marginal structures connected to it, which in turn leads to the delayed P300 latency.

Our results mean significant negative correlation between H-EPVS count and Verbal fluency, which further supports the previous research conclusions [7]. Research in Macaques found that areas similar to human temporo-parietal junction (TPJ) have two-way connections with the parahippocampal gyrus and are related to learning and memory [22]. Recent evidence suggested that the hippocampus acts as a sequence generator, extracts information from memory and participates in rapid context learning [23-25]. Verbal learning and episodic memory tests use the Ray auditory language learning test (RAVLT, immediate and delayed). We found that the AVLT immediate and delayed function decreased in the extensive H-EPVS group, but had no effect on memory function. The hippocampus was related to the process of contextual update in P300 [26]. P300 processes upstream of the hippocampus, such as the frontal cortex and TPJ, may achieve partial contextual updates. The increase of H-EPVS count may reflect focal hippocampus atrophy $[27,28]$, hippocampal atrophy was associated with verbal memory impairment, especially verbal recall delayed [29]. Extensive H-EPVS may cause the hippocampal function decrease, destroy its connection with frontal cortex, and then verbal fluency function became worse. In addition,we also found that P300 latency was negatively associated with verbal fluency function. 
Therefore, the P300 latency may be an important index for early evaluation of verbal fluency function decline in patients with lacunar stroke extensive H-EPVS.

The advantage of this study is that use objective P300ERP examination and complete neuropsychological tests. Comprehensively evaluate the potential effects of $\mathrm{H}$-EPVS on cognitive function in patients with acute lacunar stroke, which has important clinical significance.

This study also has some limitations. First of all, the sample size was relatively small, which may affect the results. Secondly, because this study only counts the H-EPVS $<3 \mathrm{~mm}$, but does not take into account the H-EPVS $>3 \mathrm{~mm}$. We did not measure hippocampal atrophy associated with H-EPVS. Finally, this study was a cross-sectional study. In follow-up studies, we will follow up to observe the long-term effects of extensive H-EPVS on P300 latency and verbal fluency function.

\section{Conclusion}

We demonstrated that the incidence of extensive H-EPVS was higher in patients with acute lacunar stroke. The P300 latency may be used as an early indicator to evaluate the verbal fluency function of extensive H-EPVS patients with acute lacunar stroke.

\section{Abbreviations}

EPVS: enlarged perivascular space; H-EPVS:enlarged perivascular space in hippocampus; ERP:eventrelated potential; EMG:evoked electromyograhic; ROC:receiver operating characteristic; BG:basal ganglia; CSO:central semioval; WMH:white matter hyperintensity; MoCA:montreal cognitive assessment; MMSE:mini-mental state examination; AVLT:auditory word learning test; DS:digit span test; SDMT:symbol digit modality test; TMT-A:trail making test-A; SCWT-A:Stroop color-word test-A; SCWT-B:Stroop color-word test-B; CSVD:cerebral small vascular disease; AUC:area under the curve; TPJ:temporo-parietal junction; Cl:confidence interval;

\section{Declarations}

\section{Acknowlegements}

We would like to thank all the participants in the study, as well as the gratitude for the cooperation of the patients and their families.

\section{Authors' contributions}

SH participated in the design, data statistical analysis, and drafting the manuscript. ZXZ and YQM helped to read and evaluated the MRI image. KKH helped with the data collection and analysis. YPF and XG helped to measure the P300 ERP. MZ and WWY were involved in the design, review, editing the manuscript and provided financial support 
Funding This study has been supported by grants from General Program of Jiangsu Commission of Health (grant number H2019051); Young Talent Development Plan of Changzhou Health Commission (CZQM2020073)

\section{Availability of data and materials}

The data that support the findings of this study are available from the corresponding author upon reasonable request.

\section{Ethics approval and consent to participate}

The study protocol was approved by the Institutional Ethics Committees of the Changzhou No.2 People's Hospital Affiliated to Nanjing Medical University (NO. 2018-KY032-01). Verbal and written informed consent was obtained from all individual participants included in the study.

\section{Consent for publication}

Not applicable.

Conflict of interest There are no conflicts of interest to declare

\section{Author details}

${ }^{1}$ Graduate School, Dalian Medical University. No.9 West Section, South Road of Lvshun, Lvshun District, Dalian 116044, Liaoning, China.

${ }^{2}$ Department of Neurology, Changzhou No.2 People's Hospital Affiliated to Nanjing Medical University. No.29, Xinglong Lane, Tianning District, Changzhou 213004, Jiangsu, China.

${ }^{3}$ Department of Psychology, Changzhou No.2 People's Hospital Affiliated to Nanjing Medical University. No.29, Xinglong Lane, Tianning District, Changzhou 213004, Jiangsu, China.

\section{References}

1. Wardlaw J M, Benveniste $H$, Nedergaard $M$, et al. Perivascular spaces in the brain: anatomy, physiology and pathology. Nat Rev Neurol. 2020; 163: 137-153; doi: 10.1038/s41582-020-0312-z.

2. Perosa V, Priester A, Ziegler G, et al. Hippocampal vascular reserve associated with cognitive performance and hippocampal volume. Brain. 2020; 1432: 622-634; doi: 10.1093/brain/awz383.

3. Marinkovi S, Milisavljevi M, Puka L. Microvascular anatomy of the hippocampal formation. Surgical Neurology. 1992; 375: 339-349; doi: 10.1016/0090-3019(92)90001-4.

4. Knight $R$, Scabini $D$. Anatomic bases of event-related potentials and their relationship to novelty detection in humans. Journal of clinical neurophysiology : official publication of the American Electroencephalographic Society. 1998; 151: 3-13; doi: 10.1097/00004691-199801000-00003. 
5. Polich J. Updating P300: an integrative theory of P3a and P3b. Clin Neurophysiol. 2007; 11810: 2128-48; doi: 10.1016/j.clinph.2007.04.019.

6. Doubal F, Maclullich A, Ferguson K, et al. Enlarged perivascular spaces on MRI are a feature of cerebral small vessel disease. Stroke. 2010; 413: 450-4; doi: 10.1161/strokeaha.109.564914.

7. Jimenez-Balado J, Riba-Llena I, Garde E, et al. Prevalence of hippocampal enlarged perivascular spaces in a sample of patients with hypertension and their relation with vascular risk factors and cognitive function. J Neurol Neurosurg Psychiatry. 2018; 896: 651-656; doi: 10.1136/jnnp-2017316724.

8. Adams H H, Cavalieri M, Verhaaren B F, et al. Rating method for dilated Virchow-Robin spaces on magnetic resonance imaging. Stroke. 2013; 446: 1732-5; doi: 10.1161/STROKEAHA.111.000620.

9. Park J H, Heo S H, Lee M H, et al. White matter hyperintensities and recurrent stroke risk in patients with stroke with small-vessel disease. Eur J Neurol. 2019; 266: 911-918; doi: 10.1111/ene.13908.

10. Blom K, Koek H L, Van Der Graaf Y, et al. Hippocampal sulcal cavities: prevalence, risk factors and association with cognitive performance. The SMART-Medea study and PREDICT-MR study. Brain Imaging Behav. 2019; 134: 1093-1102; doi: 10.1007/s11682-018-9916-y.

11. Mai L, Sposato L, Rothwell P, et al. A comparison between the MoCA and the MMSE visuoexecutive sub-tests in detecting abnormalities in TIA/stroke patients. International journal of stroke : official journal of the International Stroke Society. 2016; 114: 420-4; doi: 10.1177/1747493016632238.

12. Schmidt R, Ropele S, Ferro J, et al. Diffusion-weighted imaging and cognition in the leukoariosis and disability in the elderly study. Stroke. 2010; 415: e402-8; doi: 10.1161/strokeaha.109.576629.

13. Zhang $C$, Chen Q, Wang Y, et al. Risk factors of dilated Virchow-Robin spaces are different in various brain regions. PLoS One. 2014; 98: e105505; doi: 10.1371/journal.pone.0105505.

14. Yao M, Zhu Y C, Soumare A, et al. Hippocampal perivascular spaces are related to aging and blood pressure but not to cognition. Neurobiol Aging. 2014; 359: 2118-25; doi: 10.1016/j.neurobiolaging.2014.03.021.

15. Brown R, Benveniste $\mathrm{H}$, Black $\mathrm{S} \mathrm{E}$, et al. Understanding the role of the perivascular space in cerebral small vessel disease. Cardiovasc Res. 2018; 11411: 1462-1473; doi: 10.1093/cvr/cvy113.

16. Li Y, Li M, Yang L, et al. The relationship between blood-brain barrier permeability and enlarged perivascular spaces: a cross-sectional study. Clin Interv Aging. 2019; 14: 871-878; doi: $10.2147 /$ CIA.S204269.

17. Maclullich A M, Wardlaw J M, Ferguson $\mathrm{K} J$, et al. Enlarged perivascular spaces are associated with cognitive function in healthy elderly men. J Neurol Neurosurg Psychiatry. 2004; 7511: 1519-23; doi: 10.1136/jnnp.2003.030858.

18. Knight $R T$, Scabini $D$, Woods $D L$, et al. Contributions of temporal-parietal junction to the human auditory P3. Brain Research. 1989; 5021: 109-116; doi: 10.1016/0006-8993(89)90466-6.

19. Knight R T. Contribution of human hippocampal region to novelty detection. Nature. 1996; 3836597: 256-259; doi: 10.1038/383256a0. 
20. Picton T W. The P300 wave of the human event-related potential. J Clin Neurophysiol. 1992; 94: 45679; doi: 10.1097/00004691-199210000-00002.

21. Meszaros A, Molnar K, Nogradi B, et al. Neurovascular Inflammaging in Health and Disease. Cells. 2020; 97; doi: 10.3390/cells9071614.

22. Amaral D G, Insausti R, Cowan W M. Evidence for a direct projection from the superior temporal gyrus to the entorhinal cortex in the monkey. Brain Research. 1983; 2752: 263-277; doi: 10.1016/00068993(83)90987-3.

23. György Buzsáki, David Tingley. Space and Time: The Hippocampus as a Sequence Generator. Trends in Cognitive Sciences. 2018; doi: 10.1016/j.tics.2018.07.006.

24. Lisman J, Buzsáki G R, Eichenbaum H, et al. Viewpoints: how the hippocampus contributes to memory, navigation and cognition. Nature Neuroscience. 2017; 2011: 1434; doi: 10.1038/nn.4661.

25. Moser E I, Moser M B, Mcnaughton B L. Spatial representation in the hippocampal formation: a history. Nature Neuroence. 2017; 2011: 1448; doi: 10.1038/nn.4653.

26. Fonken $Y$ M, Kam J W Y, Knight R T. A differential role for human hippocampus in novelty and contextual processing: Implications for P300. Psychophysiology. 2020; 577: e13400; doi: 10.1111/psyp. 13400.

27. Awad, I., A., et al. Incidental subcortical lesions identified on magnetic resonance imaging in the elderly. II. Postmortem pathological correlations. Stroke. 1986; doi: 10.1161/01.str.17.6.1090.

28. Giroud M. Neurology postgraduate training: what is to be done? Journal of Neurology Neurosurgery \& Psychiatry. 2004; 7511: 1513-5; doi: 10.1136/jnnp.2004.048462.

29. Walhovd K B, Fjell A M, Reinvang I, et al. Size does matter in the long run: hippocampal and cortical volume predict recall across weeks. Neurology. 2004; 637: 1193; doi:

10.1212/01.WNL.0000140489.33249.95.

\section{Tables}

Table 1. Comparison of extensive and non-extensive H-EPVS 


\begin{tabular}{|c|c|c|c|c|}
\hline \multirow{3}{*}{ Variable } & $\begin{array}{l}\text { Non- } \\
\text { extensive }\end{array}$ & $\begin{array}{l}\text { Extensive } \mathrm{H} \text { - } \\
\text { EPVS }\end{array}$ & \multirow{3}{*}{$\begin{array}{l}t \\
\text { values } \\
\left(\chi^{2} / Z\right)\end{array}$} & \multirow[t]{3}{*}{$P$-value } \\
\hline & H-EPVS & $(n=61)$ & & \\
\hline & $\llbracket n=53 \rrbracket$ & & & \\
\hline Age, years, mean \pm SD & $64.09 \pm 5.87$ & $67.18 \pm 6.87$ & -2.558 & $0.012^{*}$ \\
\hline Male sex, $n \rrbracket \% \rrbracket$ & $43(81.1 \%)$ & $46(75.4 \%)$ & 0.542 & 0.461 \\
\hline Education years $₫$ mean $\pm S D$ & $9.87 \pm 2.77$ & $9.82 \pm 3.04$ & 0.088 & 0.930 \\
\hline Hypertension, $n \rrbracket \% \rrbracket$ & $33(62.3 \%)$ & $44(72.1 \%)$ & 1.259 & 0.262 \\
\hline Diabetes, $\mathrm{n} \rrbracket \% \rrbracket$ & $25(47.2 \%)$ & $24(39.3 \%)$ & 0.709 & 0.400 \\
\hline Hyperlipidemia, $n \llbracket \% \rrbracket$ & $16(30.2 \%)$ & $22(36.1 \%)$ & 0.441 & 0.507 \\
\hline Smoking history, $\mathrm{n} \rrbracket \% \rrbracket$ & $19(35.8 \%)$ & $30(49.2 \%)$ & 2.057 & 0.152 \\
\hline Drinking history, $\mathrm{n} \otimes \square \square$ & $13(24.5 \%)$ & $15(24.6 \%)$ & 0.000 & 0.994 \\
\hline \multicolumn{5}{|l|}{ MRI marker } \\
\hline Moderate-severe BG-EPVS, $n \rrbracket \% \rrbracket$ & $25(47.2 \%)$ & $26(42.6 \%)$ & 0.237 & 0.626 \\
\hline Moderate-severe CSO-EPVS, nヌ\%】 & $29(54.7 \%)$ & $31(50.8 \%)$ & 0.173 & 0.678 \\
\hline Moderate-severe $\mathrm{WMH}, \mathrm{n} \rrbracket \% \rrbracket$ & $19(35.8 \%)$ & $19(31.1 \%)$ & 0.282 & 0.595 \\
\hline $\begin{array}{l}\text { Presence of lacunar infarction(n) median } \\
\text { (range) }\end{array}$ & $5(2 \sim 8)$ & 7(3 9) & -1.303 & 0.192 \\
\hline MoCA, mean \pm S D & $27.23 \pm 1.10$ & $27.03 \pm 0.95$ & 1.008 & 0.316 \\
\hline Memory Z-score & $0.09 \pm 0.64$ & $-0.08 \pm 0.53$ & 1.516 & 0.132 \\
\hline AVLT immediate recall $Z$-score, mean \pm SD & $0.22 \pm 0.89$ & $-0.19 \pm 1.05$ & 2.258 & $0.026 *$ \\
\hline AVLT delayed recall Z-score, mean \pm SD & $0.25 \pm 1.02$ & $-0.22 \pm 0.94$ & 2.599 & $0.011 *$ \\
\hline AVLT recognition $Z$-score, mean \pm SD & $-0.07 \pm 1.11$ & $0.06 \pm 0.90$ & -0.720 & 0.473 \\
\hline DS Forward Z-score, mean \pm SD & $-0.05 \pm 1.04$ & $0.04 \pm 0.97$ & -0.477 & $0 . .634$ \\
\hline Information processing speed $Z$-score & $-0.13 \pm 0.45$ & $0.11 \pm 0.49$ & -2.709 & $0.008 * *$ \\
\hline SDMT Z-score, mean \pm SD & $-0.03 \pm 0.94$ & $0.03 \pm 1.06$ & -0.289 & 0.773 \\
\hline TMT-A Z-score, mean \pm SD & $-0.05 \pm 1.16$ & $0.04 \pm 0.85$ & -0.470 & 0.639 \\
\hline Stroop-A Z-score, mean \pm SD & $-0.23 \pm 0.95$ & $0.20 \pm 1.00$ & -2.319 & $0.022^{*}$ \\
\hline Stroop-B Z-score, mean \pm SD & $-0.21 \pm 0.95$ & $0.18 \pm 1.01$ & -2.117 & $0.036 *$ \\
\hline
\end{tabular}




\begin{tabular}{|llllc|}
\hline Executive function Z-score & $0.03 \pm 0.59$ & $-0.03 \pm 0.53$ & 0.587 & 0.558 \\
\hline SCWT interference Z-score, mean \pm SD & $-0.00 \pm 1.01$ & $0.00 \pm 1.00$ & -0.017 & 0.987 \\
\hline TMT interference Z-score, mean \pm SD & $0.08 \pm 0.96$ & $-0.07 \pm 1.04$ & 0.824 & 0.412 \\
\hline DS Backward Z-score, mean \pm SD & $0.02 \pm 1.05$ & $-0.02 \pm 0.97$ & 0.174 & 0.863 \\
\hline Verbal fluency Z-score, mean \pm SD & $0.47 \pm 0.54$ & $-0.41 \pm 0.50$ & 9.017 & $\mathbf{0 . 0 0 0 * *}$ \\
\hline Semantic verbal fluency Z-score, mean \pm SD & $0.56 \pm 0.83$ & $-0.49 \pm 0.87$ & 6.573 & $\mathbf{0 . 0 0 0 * *}$ \\
\hline $\begin{array}{l}\text { phonemic verbal fluency Z-score, mean } \pm \\
\text { SD }\end{array}$ & $0.38 \pm 1.02$ & $-0.33 \pm 0.86$ & 3.988 & $\mathbf{0 . 0 0 0 * *}$ \\
\hline P300 latency, mean \pm SD & $356.40 \pm 19.59$ & $393.61 \pm 30.36$ & -7.870 & $\mathbf{0 . 0 0 0 * *}$ \\
\hline P300 amplitude, mean \pm SD & $8.93 \pm 2.41$ & $8.42 \pm 2.32$ & 1.157 & 0.250 \\
\hline
\end{tabular}

Data are presented as mean \pm standard deviation, $\mathrm{n}(\%)$ or median (IQR).

$* P$ value $<0.05, * * P$ value $<0.01$

Table 2. H-EPVS count Linear regression analysis

\begin{tabular}{|lll|}
\hline \multicolumn{3}{|c|}{ P300 latency } \\
\hline & $\beta \otimes 95 \% \mathrm{Cl}$ & $P$ \\
\hline H-EPVS count & $4.659(3.870 \sim 5.448)$ & $0.000^{\star *}$ \\
\hline Age & $0.175(-0.459 \sim 0.809)$ & 0.585 \\
\hline Sex & $-2.475(-12.135 \sim 7.185)$ & 0.613 \\
\hline Education years & $0.151(-1.263 \sim 1.565)$ & 0.833 \\
\hline
\end{tabular}

** $P$ value $<0.01$

Adjust age, sex and education years

\section{Figures}




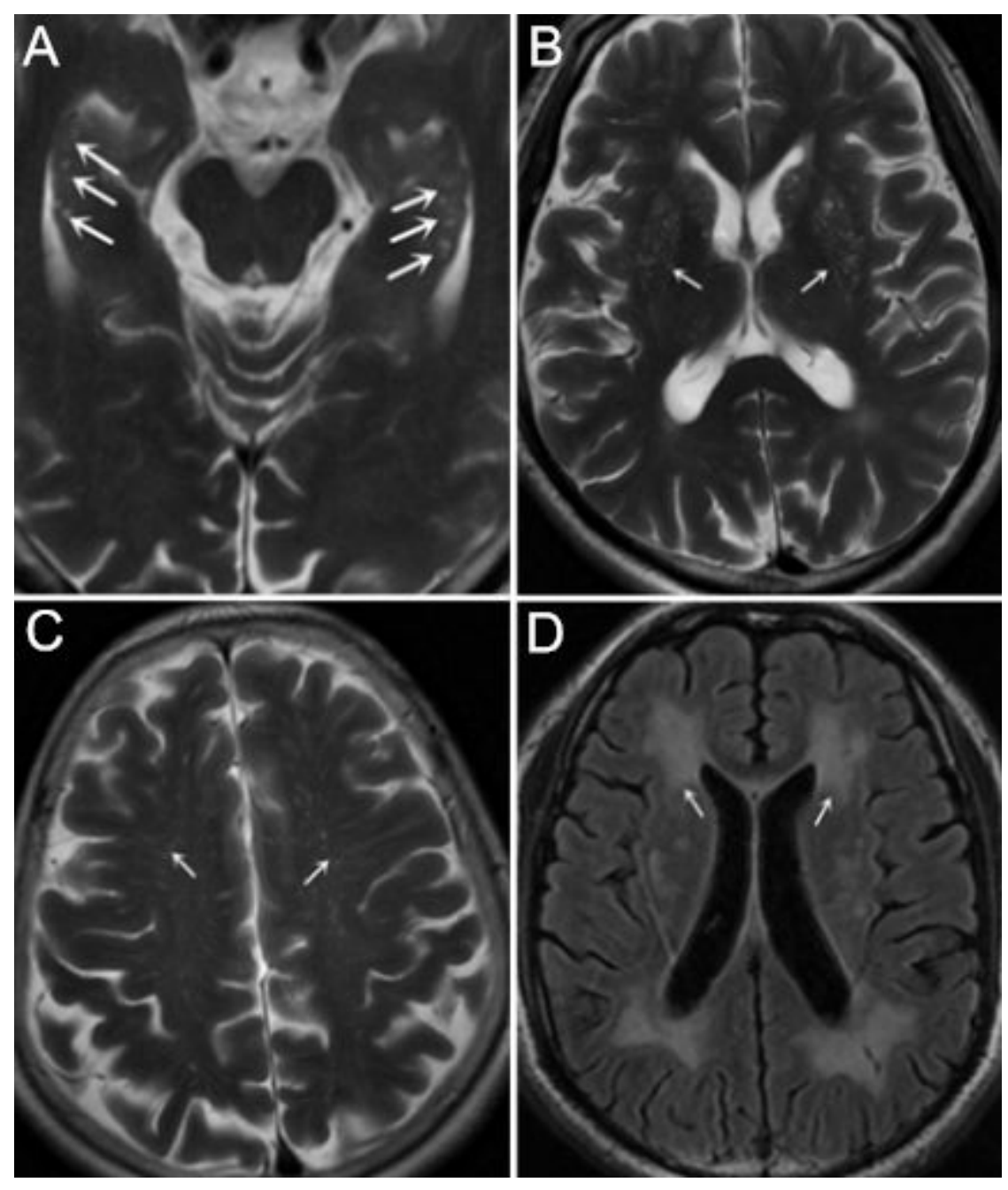

Figure 1

Figure $\mathrm{A} \sim \mathrm{C}$ is $\mathrm{T} 2 \mathrm{WI}$ image, and figureD is $\mathrm{T} 2 \mathrm{Flair}$ image. Image $\mathrm{A}$ is extensive H-EPVS; Image $\mathrm{B}$ is moderate- severe BG-EPVS; Image $\mathrm{C}$ is moderate- severe CSO-EPVS; Image $\mathrm{D}$ is moderate- severe WMH;
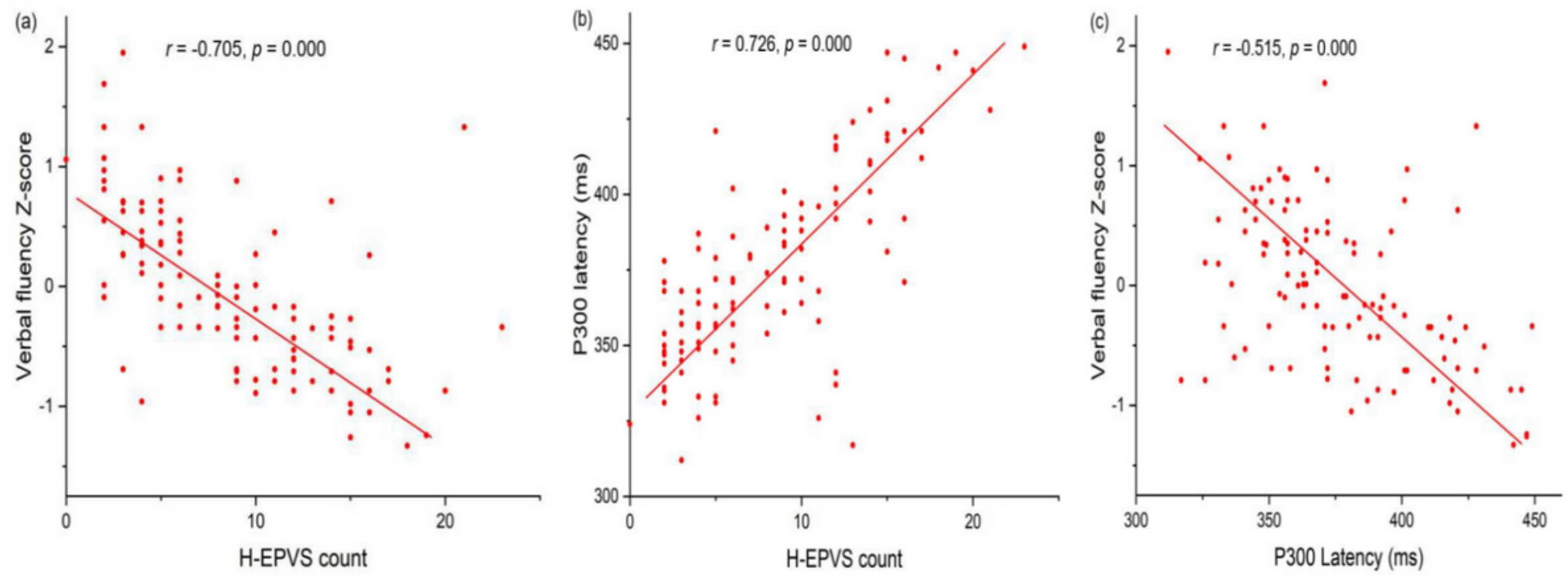

Figure 2 
Spearman correlation Analysis of H-EPVS count $(a, b)$ Correlation between H-EPVS count and verbal fluency Z-score with P300 latency (c) Correlation between verbal fluency Z-score with P300 latency

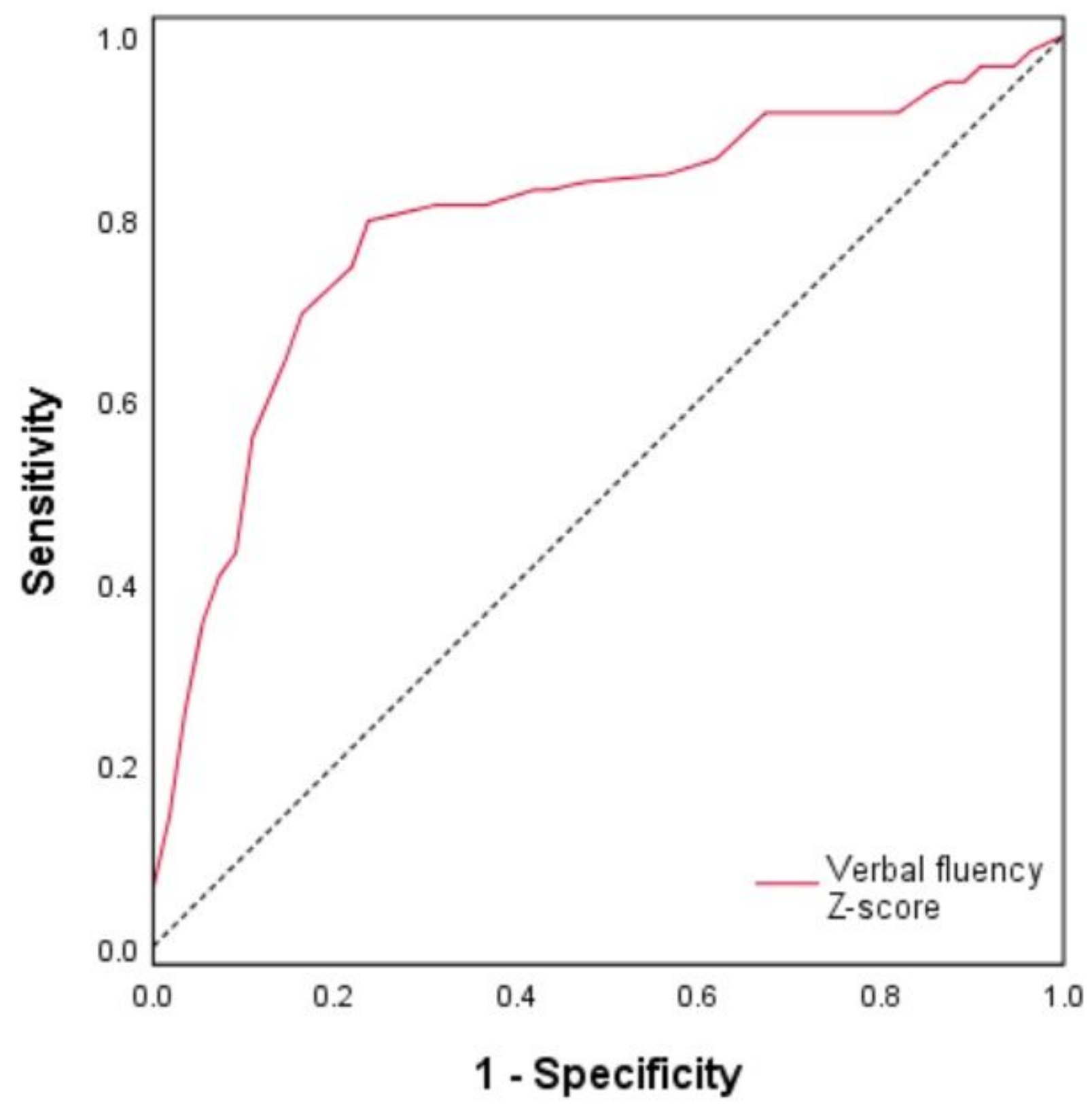

Figure 3

ROC curves of the P300 latency for predicting verbal fluency function of H-EPVS patients with lacunar stroke. The area under the curve (AUC) of P300 latency for predicting verbal fluency functional was 0.796 $(95 \% \mathrm{Cl} 0.711 \sim 0.881, \mathrm{P}<0.001)$. 